\title{
Identification of loci where DNA methylation potentially mediates genetic risk of type 1 diabetes
}

\author{
Jody $\mathrm{Ye}^{1}$, Tom G Richardson ${ }^{2}$, Wendy L McArdle ${ }^{2}$, Caroline L Relton ${ }^{2}$, Kathleen M \\ Gillespie $^{1}$, Matthew Suderman ${ }^{2}$, Gibran Hemani ${ }^{2}$
}

\begin{abstract}
Author Affiliations:
${ }^{1}$ Diabetes and Metabolism, Bristol Medical School (Translational Health Sciences), University of Bristol, Bristol, UK, BS10 5NB

${ }^{2}$ MRC Integrative Epidemiology Unit, Bristol Medical School (Population Health Sciences), University of Bristol, Bristol, UK, BS8 2BN

Corresponding to: Dr Jody Ye, Diabetes and Metabolism, Bristol Medical School,

Level 2 Learning and Research, University of Bristol, Southmead Hospital, Bristol, BS10

5NB, United Kingdom, jody.yi.ye@ bristol.ac.uk, Tel: +44 (0)117 4148041
\end{abstract}

Key words: DNA methylation, Epigenetics, Juvenile Diabetes, Causal Modeling, Single Nucleotide Polymorphism (SNP) 


\section{Abbreviations}

ARIES Accessible Resource for Integrated Epigenomic Studies

ALSPAC Avon Longitudinal Study of Parents and Children

BOX Bart's Oxford family study of Type 1 Diabetes

CpG Cytosine-phosphate-guanine dinucleotides

GWAS Genome-wide association study

HLA Human leukocyte antigen

JLIM Joint likelihood mapping

LD Linkage disequilibrium

SNP Single nucleotide polymorphism

T1D Type 1 diabetes

MAF Minor allele frequency

MR Mendelian Randomization

mQTL methylation quantitative trait loci

RA Rheumatoid arthritis

2SMR Two Sample Mendelian Randomization 


\section{Abstract}

The risk of Type 1 Diabetes (T1D) comprises both genetic and environmental components. We investigated whether genetic susceptibility to T1D could be mediated by changes in DNA methylation, an epigenetic mechanism that potentially plays a role in autoimmune diabetes. Using data from a non-diabetic population comprising blood samples taken at birth $(\mathrm{n}=844)$, childhood $(n=911)$ and adolescence $(n=907)$, we evaluated the associations between 65 top GWAS single nucleotide polymorphisms (SNPs) and genome-wide DNA methylation levels. We identified 159 proximal SNP-cytosine phosphate guanine $(\mathrm{CpG})$ pairs (cis), and 7 distal SNP-CpG associations (trans) at birth, childhood, and adolescence. There was also a systematic enrichment for methylation related SNPs to be associated with T1D across the genome, after controlling for genomic characteristics of the SNPs, implying that methylation could either be on the causal pathway to T1D or a non-causal biomarker. Combining the principles of Mendelian Randomization and genetic colocalization analysis, we provided evidence that at 5 loci, ITGB3BP, AFF3, PTPN2, CTSH and CTLA4, DNA methylation is potentially on the causal pathway to T1D.

Word count: 170 


\section{Introduction}

Type 1 diabetes (T1D) is a polygenic disease with more than $50 \%$ of genetic susceptibility attributable to the human leukocyte antigen (HLA) class II region and the remaining attributable to the non-HLA region (1). Over the last decade, genome-wide association studies identified 62 independent loci and over 100 GWAS SNPs associated with T1D risk (2), but the biological pathways for most are unknown.

DNA methylation is an epigenetic event that occurs at cytosine - phosphate - guanine (CpG) residues and can be modified by both genetic and environmental exposures. Genetic and epigenetic interactions have been postulated to contribute to susceptibility to a number of autoimmune disorders $(3 ; 4)$.

Previous work focused on identifying methylation differences among T1D monozygotic twins (5), allowing changes of DNA methylation levels that are introduced by environment to be captured (6-8), but no study has systematically investigated the causal relationship between genetically driven DNA methylation changes and T1D. Genetic variance has been reported to explains $24 \%$ of the methylation variance in childhood and $21 \%$ in middle age, when T1D can develop $(9 ; 10)$. We therefore asked whether genetic susceptibility for T1D could be mediated by DNA methylation, which subsequently lead to altered gene expression and immune cell function. To address this question, the associations between T1D susceptible single nucleotide polymorphisms (SNPs) and DNA methylation need to be established first. When a T1D-SNP is associated with DNA methylation, four potential scenarios can occur:

(1) the SNP has a causal effect on T1D mediated by the changes of DNA methylation levels (Figure 1a);

(2) the SNP has a causal effect on T1D (i.e. via altering gene expression), which in turn alters DNA methylation levels (reverse causal, Figure 1b); 
(3) the SNP that causes methylation change is in linkage disequilibrium (LD) with the causal variant of T1D (Figure 1c);

(4) the SNP influences methylation and T1D via separate mechanisms, an effect known as horizontal pleiotropy (Figure 1d).

To disentangle the causal associations between DNA methylation and T1D, we employed principles of Mendelian Randomization (MR). MR is a statistical framework to infer causal relationships in hypothesis testing, using genetic polymorphisms to create a pseudoexperimental design. Since genotypes are randomly assigned at conception and not influenced by environmental confounders, they can be used as instruments to proxy exposures that are potentially influencing a trait, thereby mimicking a randomised controlled trial (11). In the context of DNA methylation, SNPs that regulate methylation levels at nearby CpG sites (defined within $1 \mathrm{Mb}$ distance, known as cis-mQTLs) can be used to investigate the causal effect of DNA methylation on a trait (12). If a CpG site mediates genetic risk of T1D, the casual effect of this CpG site can be interpreted as the change in log odds for T1D per unit increase in the $\mathrm{CpG}$ methylation level due to its associated SNP. Compared with traditional MR, where the effects of genetic instruments on exposure and on associated traits are measured in the same population (hence one-sample MR), Two-Sample MR (2SMR) has been developed to enable causal inference using summary statistics from GWAS alone, circumventing the requirement that DNA methylation levels and T1D status are measured in the same sample, enabling much larger sample sizes (11).

Firstly, in this study, we established the association between top T1D GWAS SNPs and DNA methylation by performing an epigenome-wide association (EWAS) analysis in a young nondiabetic population, where associations are likely to be on the causal pathway to T1D. Secondly, we tested whether there is an overall association between cis-mQTLs and T1D. Subsequently, following the framework outlined in Richardson et al 2017 (13), we combined 
the principles of $2 \mathrm{SMR}$ with genetic colocalization analysis to assess the four scenarios outlined above. Finally, we tested whether the findings can be replicated in an independent cohort with T1D patients and their relatives. A flow chart summarising the analysis procedure is shown in Figure 2.

\section{Materials and methods}

\section{DNA methylation data}

Young non-diabetic population. DNA methylation data was obtained from the Avon Longitudinal Study of Parents and Children (ALSPAC) study, a large scale prospective study based in Avon, UK. ALSPAC recruited 14,541 pregnant women with expected delivery dates between $1^{\text {st }}$ April 1991 to $31^{\text {st }}$ December 1992, clinical data and biological samples were collected during pregnancy and at regular intervals postpartum from both parents and offspring $(14 ; 15)$. The study website contains details of all the data that is available through a fully searchable data dictionary http://www.bris.ac.uk/alspac/researchers/data-access/datadictionary/. DNA methylation data were generated either from whole blood or peripheral blood leukocyte samples, derived from 1,018 mother-offspring pairs using the Illumina HumanMethylation450 BeadChip ('450k array’) (Illumina, San Diego, CA, USA) as part of the Accessible Resource for Integrated Epigenomic Studies (ARIES) project (16). The array quantifies DNA methylation levels of $>485,000 \mathrm{CpG}$ sites, which covers $99 \%$ RefSeq genes (17). The ARIES participants were selected based on the availability of DNA samples at twotime points for the mother (antenatal and at follow-up when the offspring were adolescents) and three-time points for the offspring (cord blood, childhood and adolescence). Methylation data from the offspring at adolescence (mean age 17.1 years, $n=907$ ), childhood (mean age 7.5 years, $n=911$ ) and birth (mean range, $n=844$ ) were used in this study. Detailed quality 
control and normalization procedures have been described previously (16). The final methylation data contained 459,734 probes per individual.

$T 1 D$ population. As part of the methylation study, 16 families including 45 individuals were selected from the Bart's Oxford family study of type 1 diabetes (BOX) (18). DNA from probands, their parents and grandparents were analysed using the 450k array, their clinical characteristics were summarized in Supplementary Table1. In brief, DNA methylation was normalized using SWAN normalization and further corrected for batch effect, age, gender and cell heterogeneity using the Minfi and SVA package under the R statistical software (version 3.2.2), prior to plotting against genotype.

\section{Individual level genotype data}

Individual level genotype data on the ARIES cohort were generated using Illumina HumanHap550-quad chips by Sample Logistics and Genotyping Facilities at the Wellcome Trust Sanger Institute and LabCorp (Laboratory Corporation of America). Details for quality control have been described before (19). SNPs $(n=113)$ spanning 57 genomic regions that are associated with T1D at genome-wide significant level were obtained from immunobase.org, including six additional SNPs associated with T1D from a recent GWAS study (2). Among theses, sixty-seven available SNPs $\left(\mathrm{LD} \mathrm{r}^{2}<0.1\right)$ were selected and where necessary, proxy SNPs (minimal $r^{2}=0.6$ ) were used to replace the original variants in order to obtain the required odds ratios for downstream MR analysis. Genotype data of sixty-five SNPs were available from ARIES, which were summarised in Supplementary Table 2.

Genotype data for the BOX participants were determined using Taqman ${ }^{\circledR}$ allele discrimination assays (Life Technologies, UK). Taqman probes for rs2269242, rs9653442, 
rs3087243, rs3825932, and rs1893217 were purchased from Life Technologies (Thermo Fisher, UK).

\section{mQTL-CpG association analyses}

To remove outliers, bimodally distributed beta values were converted to $\mathrm{M}$ values (20) and then rank-transformed into normal distributions. We regressed 65 independent SNPs with 459,734 CpG sites measured at adolescence, childhood and birth separately using a mixed effect linear model; age, gender, cellular compositions (21) were also included as covariates in the model. A Bonferroni threshold of $1.6 \times 10^{-9}\left(0.05 / 65^{*} 465,877\right)$ was used to correct for multiple-testing. Analyses were performed using the MatrixEQTL package in $\mathrm{R}$ 3.2.2 statistical software on the University of Bristol High Performance Computing (HPC) cluster.

\section{Summary statistics}

T1D GWAS summary statistics were obtained from two meta-analyses: one for the initial analysis (Data 1) (2) and one for replication (Data 2) (22); details were summarized in Supplementary Table3. Data were retrieved from Immunobase.org; only $p$-values for Data 2 were available.

mQTL summary data was obtained from ARIES participants from a previous study (9), in which $p$-values of SNP-CpG associations were retrieved using the TwoSampleMR R package.

\section{mQTL enrichment analyses}


We investigated whether there is an overall association between cis-mQTLs and T1D more than expected by chance. cis-mQTLs $\left(\mathrm{LD} \mathrm{r}^{2}<0.1\right)$ that have shown strong associations $\left(p<1 \times 10^{-14}\right.$, Type I error rate $\left.0.2 \%\right)$ with DNA methylation were retrieved from the ARIES cohort (9); their $p$-values for T1D associations were retrieved from the two GWAS summary statistics (Data 1 and Data 2, respectively). The enrichment of cis-mQTL could be either due to their (1) distinct SNP properties (allele frequency, LD, gene distance) or (2) due to distinct genomic locations (i.e. promoter, intron, exon, 5' UTR, or 3' UTR). To control for these two factors, null SNPs were selected to match cis-mQTLs in two ways. Firstly, null SNPs were chosen based on similarities in minor allele frequency (MAF) and LD structures (23). For example, null SNPs must be at least $1000 \mathrm{~kb}$ away from mQTLs; the maximum MAF deviation of null SNPs from mQTLs is 0.02; and LD scores (24) of null SNPs are in the same quintile bin of mQTLs. Secondly, null SNPs were chosen based on similarities in genomic annotations, such as, intron, exon, 5'UTR, 3'UTR or promoter SNPs. For both methods, null SNPs were sampled without replacement. Fisher's combined probability test was used to obtain an overall association with T1D:

$$
X_{2 k}^{2} \sim-2 \sum_{i=1}^{k} \ln p_{i}
$$

To generate a distribution for null SNPs, the same number of null SNPs was randomly drawn 10,000 times to obtain 10,000 combined $p$-values. The empirical $p$-value, reflecting the likelihood of observing a combined $p$-value at least as extreme as the combined $p$-value for mQTLs in the null distribution, is calculated by ranking all the $10,000 p$-values for null SNPs (9).

\section{Bi-directional Two-Sample Mendelian Randomization}


Forward 2SMR was used to test whether a CpG is causally influencing T1D (Figure 1a). To be considered as a valid instrument to proxy DNA methylation, first, a SNP must be strongly associated with DNA methylation; second, a SNP must only influence T1D via DNA methylation; third, a SNP must be independent of confounders of the methylation-T1D associations (i.e. hyperglycemia and medications) (25). To reduce potential pleotropic effect, only cis-mQTLs were used as instruments since trans-mQTLs may influence methylation via multiple biological pathways. To exclude potential instrument-confounder associations, we also ensured that instruments were not associated with fasting glucose concentration in a large GWAS meta-analysis involving 133,010 non-diabetic European individuals (26). The causal effect of $\mathrm{CpG}$ to T1D was then determined using the Wald ratio estimator, derived by the ratio between the log odds of SNP on T1D association and the coefficient of SNP on DNA methylation association (27).

To test the causal effect of T1D on DNA methylation, multiple instruments were used for individual $\mathrm{CpG}$ as an outcome (Figure 1b). For all CpGs, mQTL used as an instrument in the forward 2SMR were excluded. The causal effects of multiple SNPs were combined in a fixed-effect meta-analysis using MR - inverse variance weighting. To account for multiple testing in the outcome (i.e. CpGs are co-methylated if they are located close to each other on a chromosome), we used matrix spectral decomposition (matSpDlite) (28) to determine the number of independent tests in the outcome, it also reports an alpha level that is required to keep the Type I Error rate at 5\% (28).

\section{MR-Steiger directionally test}


In some situations, such as DNA methylation, it is difficult to ascertain whether genetic risk first causes changes in DNA methylation which subsequently results in T1D risk or vice versa. As a verification of $2 \mathrm{SMR}$ results, we used MR-Steiger to assess whether DNA methylation is the likely exposure and T1D risk is the likely outcome. MR-Steiger estimates the proportion of variance in the exposure and in the outcome that is explained by genetic instruments. Causal direction is then determined based on whether exposure variance or outcome variance is subject to the primary effect of SNPs (29).

\section{Bivariate fine-mapping}

To estimate the probability of shared causal variants for DNA methylation and T1D (scenario 3, Figure 1c), we performed joint likelihood mapping (JLIM) (30). Concordance between top SNPs for the two traits would suggest that DNA methylation potentially reside in the causal pathway to T1D risk. The concordance rates were determined after accounting for chance, under 1000 permutations.

\section{Results}

The association between DNA methylation and T1D susceptible SNPs across three-time points of life

Of the 65 independent T1D GWAS variants, thirty-eight SNPs were consistently found to associate with a total of $166 \mathrm{CpG}$ sites under the Bonferroni corrected threshold $(p<1.6 \mathrm{e}-9)$ at adolescence, childhood and birth in the ARIES cohort. Seven SNP-CpG pairs were in trans (distance $>1 \mathrm{Mb}$ ), the remainder in cis; these data are summarised in Supplementary Table4. Figure 3 shows the genomic distribution of 166 total CpGs, including extensive associations 
at the HLA locus (Supplementary Figure1). According to 450k array annotation, approximately $45 \% \mathrm{CpGs}$ were located in the gene body / introns; $24 \%$ in promoters / distal promoters. Methylation variance $\left(\mathrm{R}^{2}\right)$ explained by T1D SNPs varied largely, the strongest association lies between rs7149271 and cg20045882 on chromosome 14, where rs7149271 explained greater than $78 \%$ methylation variance across all three-time points. At the HLA locus, rs3104163 is in high LD with rs9272346 $\left(\mathrm{r}^{2}=0.84\right)$, a variant most strongly associated with T1D but not available in our summary statistics $(\mathrm{OR}=18.5)$. rs3104363 regulates 70 CpGs within the HLA locus and the most strongly associated CpG site is $\operatorname{cg} 01889448$, for which rs3104363 explained at least 53\% methylation variance across three-time points. The overall effect sizes of T1D variants on DNA methylation levels are consistent across threetime points, where the correlation between adolescence and childhood is 0.996 (95\% CI: 0.995, 0.997, $p<2.2 \mathrm{e}-16)$; between adolescence and birth is 0.984 (95\% CI: 0.979, 0.989, $p$ $<2.2 \mathrm{e}-16$ ); and between childhood and birth is 0.987 (95\% CI: $0.982,0.990, p<2.2 \mathrm{e}-16$ ).

\section{Genome-wide enrichment of cis-mQTLs and Type 1 diabetes associations}

As a primary analysis, we overlapped adolescent cis-mQTLs with an initial discovery dataset (Data 1). These cis-mQTLs were evenly distributed across the genome (data not shown) and were devoid of HLA-SNPs (chr6: 28,477, 797-33, 448,354, hg19). Their overall associations with T1D were significantly enriched in SNPs with low GWAS $p$-values, matching null SNPs to cis-mQTLs either by SNP properties (Figure 4 a) or by genomic annotations (Figure $4 \mathrm{~b}$ ). Secondary analyses using childhood and neonatal cis-mQTLs revealed the same findings (Supplementary Figure2). To verify these observations, we performed a replication study using Data 2. Compared to Data 1, there was a stronger enrichment when cis-mQTLs were matched either by SNP properties or by genomic annotations (Supplementary Figure2). These 
data suggest that there is a shared genetic influence of DNA methylation levels and T1D. Furthermore, from each of the enrichment analyses we identified a number of cis-mQTLs that have smaller observed GWAS $p$-values than theoretical $p$-values (the probability of T1D association by chance). Most of these cis-mQTLs lie within known T1D susceptible loci and are in strong to moderate LD with index SNPs. However, rs605093 resides at Chr11q24.3 that was not reported by previous GWA studies or in LD with any index variants ( $p$ observed. meta $\left.=4.22 \mathrm{e}-6, p_{\text {theoretical }}=1.01 \mathrm{e}-5\right)$.

\section{DNA methylation potentially mediates T1D genetic risk}

After removing SNPs with harmonization issues (31), we obtained one hundred and twentyeight CpG associations unique to 33 cis-mQTLs for analyses. Forward 2SMR showed that the Wald ratios for all the $128 \mathrm{CpGs}$ were significant and the effects were consistent at adolescence, childhood and birth $(p<0.05$, Supplementary Table5).

In the reverse 2SMR, 'matSpDlite' estimated 124 independent CpGs in the outcome and a $p$ value of $4.12 \mathrm{e}-4$ is required to keep the type I error rate at $5 \%$. At this threshold, there was no evidence for reverse causation at all three-time points. It is however important to note that the statistical power to detect an effect in this direction is low because the outcome sample size was small. These data are summarised in Supplementary Table5.

\section{MR-Steiger test to verify the direction of causality}

MR-Steiger at the forward direction showed that for most CpGs within the HLA region, SNPs explained methylation variance more than T1D variance (36 out of the $64 \mathrm{CpGs}$ 
indicated methylation as the exposure consistent at three-time points, $p<0.01$, sensitivity ratio >2.35, Supplementary Table6). Outside the HLA, the same causal direction was inferred for all the CpGs and this effect was consistent at all three-time points (Supplementary Table6). We did not test the reverse direction due to insufficient statistical power to detect an effect.

\section{Bivariate fine-mapping pinpointed overlapping methylation and T1D causal variants}

In the 32 non-HLA loci, JLIM analyses pinpointed shared causal variants in 5 loci, mediated by $10 \mathrm{CpG}$ sites $(p<1 \mathrm{e}-3$, Table 1 , Figure 5). CpGs in the HLA region were however excluded from the analysis owing to the extensive LD structure and high false discovery rate (30).

\section{Replication in T1D cohort}

The 10 SNP - CpG associations were further assessed in 45 individuals participating the BOX study, where methylation 450k array data were available. Nine out of ten associations showed similar patterns comparing to the ARIES cohort, after fitting the DNA methylation levels and SNP genotype into linear regression models (Figure 6). However, given the small sample size of the replication cohort, there is insufficient power to obtain significant $p$-values for $\operatorname{cg} 05762488$ and for cg025744700 (Figure 6).

\section{Discussion}

One hypothesis of the mechanisms underlying T1D is that genetic variants alter DNA methylation levels, which in turn influence genes that are essential to immune tolerance as 
well as beta cell function, increasing the risk of T1D. To the best of our knowledge, this is the first study that systematically evaluated causal associations between methylation and T1D in a large-scale population.

Our EWAS identified widespread genetic and epigenetic interactions in known T1D susceptible loci; more than half of the T1D-SNPs were associated with proximal methylation variation in the genome, of which $40 \%$ resides at the HLA locus. More importantly, we showed genome-wide enrichment of cis-mQTLs and T1D associations in a young nondiabetic population at three-time points of life, implying that theses intrinsic methylation heterogeneities could either be causally influencing the liability of T1D or facilitate as biomarkers for islet autoimmunity development and progression.

A previous analysis based on SNP heritability however did not show significant association between cis-mQTLs and T1D (9). Narrow sense heritability of T1D was estimated to be approximately $0.8(32 ; 33)$. The lack of enrichment of cis-mQTLs in SNP heritability found by the previous study, was probably due to limited number of cis-mQTLs used in the estimation. Since approximately 20,000 cis-mQTLs were identified in the ARIES study (9), more cis-mQTLs are perhaps required to capture enough genotypic variance to explain a highly heritable condition. We found that rs605093 was associated with T1D more than expected by chance. rs605093 was detected as one of the most associated T1D SNPs but failed to reach genome-wide significance, although no follow-up study was performed to confirm its true association (34). Since GWAS tend to omit variants with small effect sizes due to the burden of multiple testing, our data may suggest a weak effect of rs605093 on T1D. This SNP is located at the intron 1 of FLI-1 (Friend leukemia integration 1 transcription 
factor), which overlaps with a known susceptible region for rheumatoid arthritis (RA) and systemic lupus erythematosus (SLE).

Given that associations between methylation and T1D are subject to confounding and reverse causation, we applied the principles of Mendelian Randomization and JLIM to evaluate their causal relationships. Although at majority of the loci, DNA methylation appeared to be noncausal, our analysis pinpointed 5 loci, where T1D risk is potentially mediated by methylation variations at $10 \mathrm{CpG}$ sites. Interrogating regulatory features from the ENCODE/Roadmap consortium revealed and enrichment of CpGs with chromosome regulatory elements (Supplementary Figure3). For example, the rs2269242 (tagging ITGB3BP) associated CpG (cg05762488) is located within a dense DNase I hypersensitive region and transcription factor binding site adjacent to gene PGMI (phosphoglucomutase -1) and upstream of the gene ITGB3BP (integrin beta 3 binding protein beta3-endonexin). ITGB3BP is a new candidate gene for T1D (2) and is involved in signalling pathways of apoptosis (35). The two CpGs (cg06183267 and cg07349094) associated with rs9653442 are situated in the exon1 of AFF3 (AF4/FMR2 Family Member 3), which is a region enriched with DNase I hypersensitivity and $\mathrm{H} 3 \mathrm{~K} 4 \mathrm{me} 3$ (associates with enhancers) signals in a range of immune cells. AFF3 is a risk gene for rheumatoid arthritis (RA) (36), juvenile idiopathic arthritis (37) and T1D $(2 ; 38)$ and may be involved in lymphoid development and plasma cell differentiation (39; 40). The two CpGs (cg25744700 and cg18738367) associated with rs3825932 are located in intron 1 and 5' upstream of the gene CTSH (Cathepsin H), respectively. cg25744700 overlaps with a H3K27Ac peak (marks active chromatin), a DNase I cluster, as well as a transcription factor binding region; cg18738367 co-localizes with a DNase I cluster. Lowered gene expression of CTSH in beta cells has been correlated with increased beta cell 
apoptosis upon cytokine exposure as well as faster diabetes progression (41). The detailed functional effects of these methylation variations require follow-up laboratory investigations.

To support our data, we observed similar association in 9 out of 10 SNP-CpG pairs in the independent BOX cohort containing T1D probands and their relatives. Although the sample size of this cohort is small $(n=45)$ and there is a wide age range in the participants, the high replication rate in methylation patterns warrants our initial observations in ARIES.

There are several limitations of our study. First, given single genetic instruments used in the forward $2 \mathrm{SMR}$, we were unable to robustly distinguish mediatory effect from horizontal pleiotropy (scenario 4, Figure 1d) without further support from experimental proof. Second, MR and its extension MR-Steiger test both suffer from insufficient statistical power to detect an effect in the reverse direction. This is because the sample size available for SNP effects on CpG levels was small (approximately 1000 individuals in the ARIES participants per time point). Third, although there is a strong evidence of shared causal variants for methylation and T1D at 5 loci, JLIM does not report which SNPs are the shared causal variants. Fourth, all our analyses were performed using whole blood or peripheral blood lymphocyte samples. As the mQTL effects are likely to be tissue and cell type specific (42), attempts to interpret our findings in a different tissue must be conducted with caution. Another limitation is that the $450 \mathrm{k}$ array typically covers $2 \%$ of the entire epigenome (17) and we were unable to investigate causal effects of uncovered $\mathrm{CpG}$ sites. It is thus possible that methylation influences T1D risk beyond the 5 loci identified in this study; those effects could not be tested without large-scale methylation sequencing data. We also reported mQTLs that regulate DNA methylation consistently at birth, childhood and adolescence, covering the 
spectrum of life when diagnosis of T1D peaks. It might be possible that some mediatory effects of DNA methylation are only specific to a particular time point (9). These changes are beyond the scope of the current analyses, future studies may focus on investigating how epigenetic regulations change during immune development.

In the HLA region, we observed extensive methylation associations with a top GWAS variant rs3104363 (T1D OR=3.7). A recent study reported over $180 \mathrm{CpGs}$ in the HLA region that were differentially methylated dependent on the T1D susceptible DQ2/DQ8 haplotype (43). Our focus was on SNPs, but 17 haplotype dependent CpGs were also strongly associated with rs3104363 in our data. Of these 13 CpGs demonstrated evidence of mediating T1D risk. However, due to high false positive rates at the HLA locus we could not use JLIM to exclude LD confounding effects (30). Functional approaches are required to better understand the effects of SNP and haplotype dependent methylation on HLA expression.

In conclusion, the identification of putative genetically driven DNA methylation changes provides a rich source for follow-up verifications, as dissecting the functional effect of genetic and epigenetic interactions may help uncover novel mechanisms that contribute to the risk of T1D development.

\section{Acknowledgement}

We are extremely grateful to all the families who took part in this study, the midwives for their help in recruiting them, and the whole ALSPAC team, which includes interviews, computer and laboratory technicians, clerical workers, research scientists, volunteers, 
managers, receptionists and nurses. We also sincerely appreciate the participants of the BOX study, nurses, administrators, and laboratory technicians for their contributions. The authors are grateful for Prof. John A Todd and Mr. Jamie Inshaw who kindly provided T1D GWAS summary statistics, as well as Prof. Yaron Tomer for his valuable advice on methylation data interpretation.

\section{Web Resources}

Matrix eQTL http://www.bios.unc.edu/research/genomic software/Matrix eQTL/;

2SMR and MR-Steiger https://github.com/MRCIEU/TwoSampleMR;

mQTL enrichment analysis https://github.com/olegkagan/Ye-et-al.-2017;

JLIM https://github.com/cotsapaslab/jlim;

matSpDlite

https://github.com/snewhouse/BRC_MH_Bioinformatics/blob/master/misc_sh/matSpDlite.R;

Immunobase www.immunobase.org;

UCSC genome browser https://genome.ucsc.edu/

\section{Research funding}

The UK medical Research Council and Wellcome (Grant ref: 102215/2/13/2) and the University of Bristol provide core support for ALSPAC. GWAS data were generated by Sample Logistics and Genotyping Facilities at the Wellcome Trust Sanger Institute and LabCorp (Laboratory Corporation of America) with support from 23andMe. Methylation data 
in the ALSPAC cohort were generated as part of the UK BBSRC-funded (BB/I025751/1 and BB/I025263/1) Accessible Resource for Integrated Epigenomic Studies (ARIES). Methylation data used in the Bart's Oxford Study (BOX) was generated using funding from Diabetes UK (14/0004869). This publication is the work of the authors; and J.Y will serve as guarantor for the contents of this paper. J.Y. was funded by a Diabetes Wellness \& Research Foundation non-clinical fellowship N-C/2016/Ye. T.G.R. was supported by the Elizabeth Blackwell Institute Proximity to Discovery award EBI 424. M.S. was supported by the Economics and social research council ES/N000498/1. G.H. was supported by the Medical Research Council MC_UU_12013/1-9. 


\begin{tabular}{|c|c|c|c|c|c|c|c|c|}
\hline SNP & Effect allele & Candidate gene & CpG ID & CpG position & CpG effect (beta $\pm S E)$ & T1D effect (beta $\pm S E)$ & 2SMR effect & 2SMR $p$-value \\
\hline rs2269242 & A & ITGB3BP,PGM1 & $\operatorname{cg} 05762488$ & Chr1: 64140332 & $0.059(0.006)$ & $0.168(0.030)$ & $2.843(0.508)$ & $2.14 \mathrm{E}-08$ \\
\hline rs9653442 & $\mathrm{C}$ & AFF3 & cg06183267 & Chr2: 100759134 & $-0.100(0.006)$ & $0.124(0.025)$ & $-1.236(0.250)$ & $7.05 \mathrm{E}-07$ \\
\hline rs9653442 & $\mathrm{C}$ & AFF3 & cg07349094 & Chr2: 100759014 & $-0.157(0.009)$ & $0.124(0.025)$ & $-0.791(0.160)$ & $7.05 \mathrm{E}-07$ \\
\hline rs3087243 & A & CTLA4 & cg22572158 & Chr2: 204731068 & $-0.070(0.008)$ & $-0.178(0.025)$ & $2.534(0.356)$ & $1.08 \mathrm{E}-12$ \\
\hline rs3825932 & $\mathrm{T}$ & $\mathrm{CTSH}$ & $\operatorname{cg} 25744700$ & Chr15: 79237217 & $-0.204(0.012)$ & $-0.141(0.030)$ & $0.692(0.147)$ & $2.60 \mathrm{E}-06$ \\
\hline rs3825932 & $\mathrm{T}$ & CTSH & cg18738367 & Chr15: 79238723 & $0.093(0.006)$ & $-0.141(0.030)$ & $-1.523(0.324)$ & $2.60 \mathrm{E}-06$ \\
\hline rs1893217 & G & PTPN2 & cg09945482 & Chr18: 12777974 & $-0.188(0.016)$ & $0.250(0.032)$ & $-1.331(0.170)$ & $5.61 \mathrm{E}-15$ \\
\hline rs1893217 & G & PTPN2 & $\operatorname{cg} 23544223$ & Chr18: 12777786 & $-0.175(0.011)$ & $0.250(0.032)$ & $-1.428(0.183)$ & $5.61 \mathrm{E}-15$ \\
\hline rs1893217 & G & PTPN2 & $\operatorname{cg} 23598886$ & Chr18: 12777645 & $-0.414(0.023)$ & $0.250(0.032)$ & $-0.604(0.077)$ & $5.61 \mathrm{E}-15$ \\
\hline rs1893217 & G & PTPN2 & cg24737193 & Chr18: 12778029 & $-0.223(0.159)$ & $0.250(0.032)$ & $-1.123(0.144)$ & $5.61 \mathrm{E}-15$ \\
\hline
\end{tabular}

Table 1: Representative 2SMR results from the adolescence dataset that survived the Joint Likelihood Mapping. CpG effect denotes the addition of effect allele relative to other allele on $\mathrm{CpG}$ methylation changes (beta coefficient $\pm \mathrm{SE}$ ); T1D effect denotes the addition of effect allele relative to other allele on T1D risk (beta coefficient \pm SE, beta coefficient equals log odds ratio); 2SMR effect denotes the change of log odds on T1D per unit increase in DNA methylation due to its associated SNP. 


\section{Figure legend}

Figure 1: Four possible scenarios that could explain the associations between SNP, DNA methylation and T1D.

a, DNA methylation mediates the genetic risk of T1D; b, SNPs first increases T1D liability, which in turn changes DNA methylation levels; c, a SNP that regulates DNA methylation could simply be in LD with another causal variant that influences T1D; d, a SNP is associated with DNA methylation and T1D via independent biological pathways (horizontal pleiotropy).

Figure 2: Flow chart summarising the overall analysis procedure in this study.

EWAS: epigenome wide association analysis; DNAm: DNA methylation; mQTL:

methylation quantitative trait loci

Figure 3: Genomic distribution of CpG sites that are associated with T1D GWAS variants.

Manhattan plot showing the $\mathrm{CpG}$ sites associated with 38 T1D GWAS variants above the Bonferroni threshold 1.6e-9 (redline); green highlighted dots were those $(n=166)$ that were consistently detected at adolescence, childhood and birth; there is a peak reflecting mQTLCpG association at the HLA locus (chromosome 6).

Figure 4: cis-mQTLs are enriched in SNPs with low GWAS $p$-values associated with T1D. 
a, A representative plot showing the enrichment analysis conducted using the adolescent data, when null SNPs were matched to cis-mQTLs via SNP properties; $b$, when null SNPs were matched to cis-mQTLs via genomic annotations. T1D GWAS $p$-values were extracted from meta-analysis Data 1.

Figure 5: DNA methylation levels of CpG sites and their associations with T1D GWAS variants that survived JLIM analyses, obtained from the ARIES adolescent participants.

$\mathrm{Y}$ - axis represents beta values for each $\mathrm{CpG}$ site. The inner most genotype in $\mathrm{X}$ - axis is comprised of two other alleles, the outer most genotype is comprised of two effect alleles.

Figure 6: Replication of SNP and DNA methylation associations in the Bart's oxford T1D cohort.

45 individuals were analysed in the BOX cohort. Nine out of ten SNP-CpG pairs showed similar associations compared to the ARIES participants. The SNP - CpG pair that did not replicate the ARIES result was highlighted in red. 


\section{References}

1. Mehers KL, Gillespie KM: The genetic basis for type 1 diabetes. Br Med Bull 2008;88:115-129

2. Cooper NJ, Wallace C, Burren OS, Cutler A, Walker N, Todd JA: Type 1 diabetes genome-wide association analysis with imputation identifies five new risk regions. bioRxiv 2017;

3. Liu Y, Aryee MJ, Padyukov L, Fallin MD, Hesselberg E, Runarsson A, Reinius L, Acevedo N, Taub M, Ronninger M, Shchetynsky K, Scheynius A, Kere J, Alfredsson L, Klareskog L, Ekstrom TJ, Feinberg AP: Epigenome-wide association data implicate DNA methylation as an intermediary of genetic risk in rheumatoid arthritis. Nat Biotechnol 2013;31:142-147

4. Farh KK, Marson A, Zhu J, Kleinewietfeld M, Housley WJ, Beik S, Shoresh N, Whitton H, Ryan RJ, Shishkin AA, Hatan M, Carrasco-Alfonso MJ, Mayer D, Luckey CJ, Patsopoulos NA, De Jager PL, Kuchroo VK, Epstein CB, Daly MJ, Hafler DA, Bernstein BE: Genetic and epigenetic fine mapping of causal autoimmune disease variants. Nature 2015;518:337-343

5. Castillo-Fernandez JE, Spector TD, Bell JT: Epigenetics of discordant monozygotic twins: implications for disease. Genome Med 2014;6:60

6. Stefan $\mathrm{M}$, Zhang $\mathrm{W}$, Concepcion $\mathrm{E}, \mathrm{Yi} Z$, Tomer Y: DNA methylation profiles in type 1 diabetes twins point to strong epigenetic effects on etiology. J Autoimmun 2014;50:33-37

7. Paul DS, Teschendorff AE, Dang MA, Lowe R, Hawa MI, Ecker S, Beyan H, Cunningham S, Fouts AR, Ramelius A, Burden F, Farrow S, Rowlston S, Rehnstrom K, Frontini M, Downes K, Busche S, Cheung WA, Ge B, Simon MM, Bujold D, Kwan T, Bourque G, Datta A, Lowy E, Clarke L, Flicek P, Libertini E, Heath S, Gut M, Gut IG, Ouwehand WH, Pastinen T, Soranzo N, Hofer SE, Karges B, Meissner T, Boehm BO, Cilio C, Elding Larsson H, Lernmark A, Steck AK, Rakyan VK, Beck S, Leslie RD: Increased DNA methylation variability in type 1 diabetes across three immune effector cell types. Nat Commun 2016;7:13555

8. Elboudwarej E, Cole M, Briggs FB, Fouts A, Fain PR, Quach H, Quach D, Sinclair E, Criswell LA, Lane JA, Steck AK, Barcellos LF, Noble JA: Hypomethylation within gene promoter regions and type 1 diabetes in discordant monozygotic twins. J Autoimmun 2016;68:23-29

9. Gaunt TR, Shihab HA, Hemani G, Min JL, Woodward G, Lyttleton O, Zheng J, Duggirala A, McArdle WL, Ho K, Ring SM, Evans DM, Davey Smith G, Relton CL: Systematic identification of genetic influences on methylation across the human life course. Genome Biol 2016;17:61

10. Thomas NJ, Jones SE, Weedon MN, Shields BM, Oram RA, Hattersley AT: Frequency and phenotype of type 1 diabetes in the first six decades of life: a cross-sectional, genetically stratified survival analysis from UK Biobank. Lancet Diabetes Endocrinol 2017;

11. Davey Smith G, Hemani G: Mendelian randomization: genetic anchors for causal inference in epidemiological studies. Hum Mol Genet 2014;23:R89-98

12. Relton CL, Davey Smith G: Two-step epigenetic Mendelian randomization: a strategy for establishing the causal role of epigenetic processes in pathways to disease. Int J Epidemiol 2012;41:161-176

13. Richardson TG, Zheng J, Davey Smith G, Timpson NJ, Gaunt TR, Relton CL, Hemani G: Causal epigenome-wide association study identifies $\mathrm{CPG}$ sites that influence cardiovascular disease risk. bioRxiv 2017;

14. Boyd A, Golding J, Macleod J, Lawlor DA, Fraser A, Henderson J, Molloy L, Ness A, Ring S, Smith GD: Cohort Profile: The 'Children of the 90s'-the index offspring of the Avon Longitudinal Study of Parents and Children. International Journal of Epidemiology 2013;42:111-127

15. Fraser A, Macdonald-Wallis C, Tilling K, Boyd A, Golding J, Smith GD, Henderson J, Macleod J, Molloy L, Ness A, Ring S, Nelson SM, Lawlor DA: Cohort Profile: The Avon Longitudinal Study of Parents and Children: ALSPAC mothers cohort. International Journal of Epidemiology 2013;42:97-110 16. Relton CL, Gaunt T, McArdle W, Ho K, Duggirala A, Shihab H, Woodward G, Lyttleton O, Evans DM, Reik W, Paul YL, Ficz G, Ozanne SE, Wipat A, Flanagan K, Lister A, Heijmans BT, Ring SM, Davey 
Smith G: Data Resource Profile: Accessible Resource for Integrated Epigenomic Studies (ARIES). Int J Epidemiol 2015;44:1181-1190

17. Bibikova M, Barnes B, Tsan C, Ho V, Klotzle B, Le JM, Delano D, Zhang L, Schroth GP, Gunderson $\mathrm{KL}$, Fan JB, Shen R: High density DNA methylation array with single $\mathrm{CpG}$ site resolution. Genomics 2011;98:288-295

18. Gardner SG, Bingley PJ, Sawtell PA, Weeks S, Gale EA: Rising incidence of insulin dependent diabetes in children aged under 5 years in the Oxford region: time trend analysis. The Bart's-Oxford Study Group. BMJ 1997;315:713-717

19. Elliott HR, Shihab HA, Lockett GA, Holloway JW, McRae AF, Smith GD, Ring SM, Gaunt TR, Relton CL: Role of DNA Methylation in Type 2 Diabetes Etiology: Using Genotype as a Causal Anchor. Diabetes 2017;66:1713-1722

20. Du $P$, Zhang $X$, Huang CC, Jafari N, Kibbe WA, Hou L, Lin SM: Comparison of Beta-value and $M-$ value methods for quantifying methylation levels by microarray analysis. BMC Bioinformatics 2010;11:587

21. Houseman EA, Accomando WP, Koestler DC, Christensen BC, Marsit CJ, Nelson HH, Wiencke JK, Kelsey KT: DNA methylation arrays as surrogate measures of cell mixture distribution. BMC Bioinformatics 2012;13:86

22. Bradfield JP, Qu HQ, Wang K, Zhang H, Sleiman PM, Kim CE, Mentch FD, Qiu H, Glessner JT, Thomas KA, Frackelton EC, Chiavacci RM, Imielinski M, Monos DS, Pandey R, Bakay M, Grant SF, Polychronakos $\mathrm{C}$, Hakonarson $\mathrm{H}$ : A genome-wide meta-analysis of six type 1 diabetes cohorts identifies multiple associated loci. PLoS Genet 2011;7:e1002293

23. Bulik-Sullivan B, Finucane HK, Anttila V, Gusev A, Day FR, Loh PR, ReproGen C, Psychiatric Genomics C, Genetic Consortium for Anorexia Nervosa of the Wellcome Trust Case Control C, Duncan L, Perry JR, Patterson N, Robinson EB, Daly MJ, Price AL, Neale BM: An atlas of genetic correlations across human diseases and traits. Nat Genet 2015;47:1236-1241

24. Bulik-Sullivan BK, Loh PR, Finucane HK, Ripke S, Yang J, Schizophrenia Working Group of the Psychiatric Genomics C, Patterson N, Daly MJ, Price AL, Neale BM: LD Score regression distinguishes confounding from polygenicity in genome-wide association studies. Nat Genet 2015;47:291-295 25. Burgess S, Scott RA, Timpson NJ, Davey Smith G, Thompson SG, Consortium E-I: Using published data in Mendelian randomization: a blueprint for efficient identification of causal risk factors. Eur J Epidemiol 2015;30:543-552

26. Scott RA, Lagou V, Welch RP, Wheeler E, Montasser ME, Luan J, Magi R, Strawbridge RJ, Rehnberg E, Gustafsson S, Kanoni S, Rasmussen-Torvik LJ, Yengo L, Lecoeur C, Shungin D, Sanna S, Sidore C, Johnson PC, Jukema JW, Johnson T, Mahajan A, Verweij N, Thorleifsson G, Hottenga JJ, Shah S, Smith AV, Sennblad B, Gieger C, Salo P, Perola M, Timpson NJ, Evans DM, Pourcain BS, Wu Y, Andrews JS, Hui J, Bielak LF, Zhao W, Horikoshi M, Navarro P, Isaacs A, O'Connell JR, Stirrups K, Vitart V, Hayward C, Esko T, Mihailov E, Fraser RM, Fall T, Voight BF, Raychaudhuri S, Chen H, Lindgren CM, Morris AP, Rayner NW, Robertson N, Rybin D, Liu CT, Beckmann JS, Willems SM, Chines PS, Jackson AU, Kang HM, Stringham HM, Song K, Tanaka T, Peden JF, Goel A, Hicks AA, An P, Muller-Nurasyid M, Franco-Cereceda A, Folkersen L, Marullo L, Jansen $H$, Oldehinkel AJ, Bruinenberg $M$, Pankow JS, North KE, Forouhi NG, Loos RJ, Edkins S, Varga TV, Hallmans G, Oksa H, Antonella M, Nagaraja R, Trompet S, Ford I, Bakker SJ, Kong A, Kumari M, Gigante B, Herder C, Munroe PB, Caulfield M, Antti J, Mangino M, Small K, Miljkovic I, Liu Y, Atalay M, Kiess W, James AL, Rivadeneira F, Uitterlinden AG, Palmer CN, Doney AS, Willemsen G, Smit JH, Campbell S, Polasek O, Bonnycastle LL, Hercberg S, Dimitriou M, Bolton JL, Fowkes GR, Kovacs P, Lindstrom J, Zemunik T, Bandinelli S, Wild SH, Basart HV, Rathmann W, Grallert H, Replication DIG, Meta-analysis C, Maerz W, Kleber ME, Boehm BO, Peters A, Pramstaller PP, Province MA, Borecki IB, Hastie ND, Rudan I, Campbell H, Watkins H, Farrall M, Stumvoll M, Ferrucci L, Waterworth DM, Bergman RN, Collins FS, Tuomilehto J, Watanabe RM, de Geus EJ, Penninx BW, Hofman A, Oostra BA, Psaty BM, Vollenweider P, Wilson JF, Wright AF, Hovingh GK, Metspalu A, Uusitupa M, Magnusson PK, Kyvik KO, Kaprio J, Price JF, Dedoussis GV, Deloukas P, Meneton P, Lind L, Boehnke M, Shuldiner AR, van Duijn CM, Morris AD, Toenjes A, 
Peyser PA, Beilby JP, Korner A, Kuusisto J, Laakso M, Bornstein SR, Schwarz PE, Lakka TA, Rauramaa R, Adair LS, Smith GD, Spector TD, Illig T, de Faire U, Hamsten A, Gudnason V, Kivimaki M, Hingorani A, Keinanen-Kiukaanniemi SM, Saaristo TE, Boomsma DI, Stefansson K, van der Harst P, Dupuis J, Pedersen NL, Sattar N, Harris TB, Cucca F, Ripatti S, Salomaa V, Mohlke KL, Balkau B, Froguel P, Pouta A, Jarvelin MR, Wareham NJ, Bouatia-Naji N, McCarthy MI, Franks PW, Meigs JB, Teslovich TM, Florez JC, Langenberg C, Ingelsson E, Prokopenko I, Barroso I: Large-scale association analyses identify new loci influencing glycemic traits and provide insight into the underlying biological pathways. Nat Genet 2012;44:991-1005

27. Haycock PC, Burgess S, Wade KH, Bowden J, Relton C, Davey Smith G: Best (but oft-forgotten) practices: the design, analysis, and interpretation of Mendelian randomization studies. Am J Clin Nutr 2016;103:965-978

28. Nyholt DR: A simple correction for multiple testing for single-nucleotide polymorphisms in linkage disequilibrium with each other. Am J Hum Genet 2004;74:765-769

29. Hemani G, Tilling K, Davey Smith G: Orienting the causal relationship between imprecisely measured traits using GWAS summary data. PLoS Genet 2017;13:e1007081

30. Chun S, Casparino A, Patsopoulos NA, Croteau-Chonka DC, Raby BA, De Jager PL, Sunyaev SR, Cotsapas C: Limited statistical evidence for shared genetic effects of eQTLs and autoimmunedisease-associated loci in three major immune-cell types. Nat Genet 2017;49:600-605 31. Hartwig FP, Davies NM, Hemani G, Davey Smith G: Two-sample Mendelian randomization: avoiding the downsides of a powerful, widely applicable but potentially fallible technique. Int J Epidemiol 2016;45:1717-1726

32. Hyttinen V, Kaprio J, Kinnunen L, Koskenvuo M, Tuomilehto J: Genetic liability of type 1 diabetes and the onset age among 22,650 young Finnish twin pairs: a nationwide follow-up study. Diabetes 2003;52:1052-1055

33. Li YR, Zhao SD, Li J, Bradfield JP, Mohebnasab M, Steel L, Kobie J, Abrams DJ, Mentch FD, Glessner JT, Guo Y, Wei Z, Connolly JJ, Cardinale CJ, Bakay M, Li D, Maggadottir SM, Thomas KA, Qui $H$, Chiavacci RM, Kim CE, Wang F, Snyder J, Flato B, Forre O, Denson LA, Thompson SD, Becker ML, Guthery SL, Latiano A, Perez E, Resnick E, Strisciuglio C, Staiano A, Miele E, Silverberg MS, Lie BA, Punaro M, Russell RK, Wilson DC, Dubinsky MC, Monos DS, Annese V, Munro JE, Wise C, Chapel H, Cunningham-Rundles C, Orange JS, Behrens EM, Sullivan KE, Kugathasan S, Griffiths AM, Satsangi J, Grant SF, Sleiman PM, Finkel TH, Polychronakos C, Baldassano RN, Luning Prak ET, Ellis JA, Li H, Keating BJ, Hakonarson $\mathrm{H}$ : Genetic sharing and heritability of paediatric age of onset autoimmune diseases. Nat Commun 2015;6:8442

34. Cooper JD, Smyth DJ, Smiles AM, Plagnol V, Walker NM, Allen JE, Downes K, Barrett JC, Healy BC, Mychaleckyj JC, Warram JH, Todd JA: Meta-analysis of genome-wide association study data identifies additional type 1 diabetes risk loci. Nat Genet 2008;40:1399-1401

35. Li D, Das S, Yamada T, Samuels HH: The NRIF3 family of transcriptional coregulators induces rapid and profound apoptosis in breast cancer cells. Mol Cell Biol 2004;24:3838-3848

36. Barton A, Eyre S, Ke X, Hinks A, Bowes J, Flynn E, Martin P, Consortium Y, Consortium B, Wilson AG, Morgan AW, Emery P, Steer S, Hocking LJ, Reid DM, Harrison P, Wordsworth P, Thomson W, Worthington J: Identification of AF4/FMR2 family, member 3 (AFF3) as a novel rheumatoid arthritis susceptibility locus and confirmation of two further pan-autoimmune susceptibility genes. Hum Mol Genet 2009;18:2518-2522

37. Hinks A, Eyre S, Ke X, Barton A, Martin P, Flynn E, Packham J, Worthington J, Childhood Arthritis Prospective S, Consortium U, Group BS, Thomson W: Association of the AFF3 gene and IL2/IL21 gene region with juvenile idiopathic arthritis. Genes Immun 2010;11:194-198

38. Onengut-Gumuscu S, Chen WM, Burren O, Cooper NJ, Quinlan AR, Mychaleckyj JC, Farber E, Bonnie JK, Szpak M, Schofield E, Achuthan P, Guo H, Fortune MD, Stevens H, Walker NM, Ward LD, Kundaje A, Kellis M, Daly MJ, Barrett JC, Cooper JD, Deloukas P, Type 1 Diabetes Genetics C, Todd JA, Wallace $C$, Concannon P, Rich SS: Fine mapping of type 1 diabetes susceptibility loci and evidence for colocalization of causal variants with lymphoid gene enhancers. Nat Genet 2015;47:381-386 
bioRxiv preprint doi: https://doi.org/10.1101/248260; this version posted February 8, 2018. The copyright holder for this preprint (which was not certified by peer review) is the author/funder. All rights reserved. No reuse allowed without permission.

39. Ma C, Staudt LM: LAF-4 encodes a lymphoid nuclear protein with transactivation potential that is homologous to AF-4, the gene fused to MLL in $t(4 ; 11)$ leukemias. Blood 1996;87:734-745

40. Minnich M, Tagoh H, Bonelt P, Axelsson E, Fischer M, Cebolla B, Tarakhovsky A, Nutt SL, Jaritz M, Busslinger M: Multifunctional role of the transcription factor Blimp-1 in coordinating plasma cell differentiation. Nat Immunol 2016;17:331-343

41. Floyel T, Brorsson C, Nielsen LB, Miani M, Bang-Berthelsen $C H$, Friedrichsen $M$, Overgaard AJ, Berchtold LA, Wiberg A, Poulsen P, Hansen L, Rosinger S, Boehm BO, Ram R, Nguyen Q, Mehta M, Morahan G, Concannon P, Bergholdt R, Nielsen JH, Reinheckel T, von Herrath M, Vaag A, Eizirik DL, Mortensen HB, Storling J, Pociot F: CTSH regulates beta-cell function and disease progression in newly diagnosed type 1 diabetes patients. Proc Natl Acad Sci U S A 2014;111:10305-10310

42. Gutierrez-Arcelus $M$, Ongen $H$, Lappalainen $T$, Montgomery SB, Buil A, Yurovsky A, Bryois J, Padioleau I, Romano L, Planchon A, Falconnet E, Bielser D, Gagnebin M, Giger T, Borel C, Letourneau A, Makrythanasis P, Guipponi M, Gehrig C, Antonarakis SE, Dermitzakis ET: Tissue-specific effects of genetic and epigenetic variation on gene regulation and splicing. PLoS Genet 2015;11:e1004958 43. Kindt ASD, Fuerst RW, Knoop J, Laimighofer M, Telieps T, Hippich M, Woerheide MA, Wahl S, Wilson R, Sedlmeier EM, Hommel A, Todd JA, Krumsiek J, Ziegler AG, Bonifacio E: Allele-specific methylation of type 1 diabetes susceptibility genes. J Autoimmun 2017; 
a

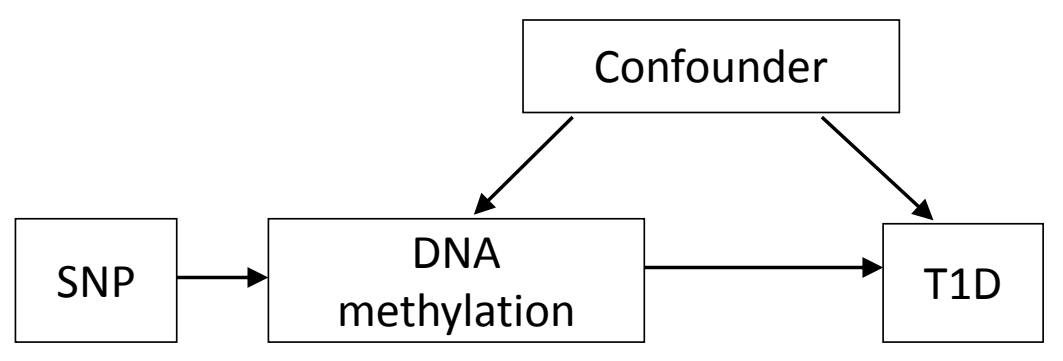

C

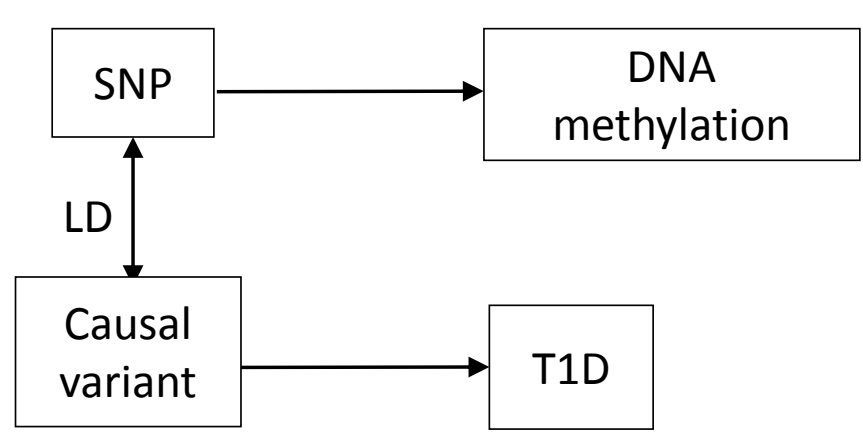

b

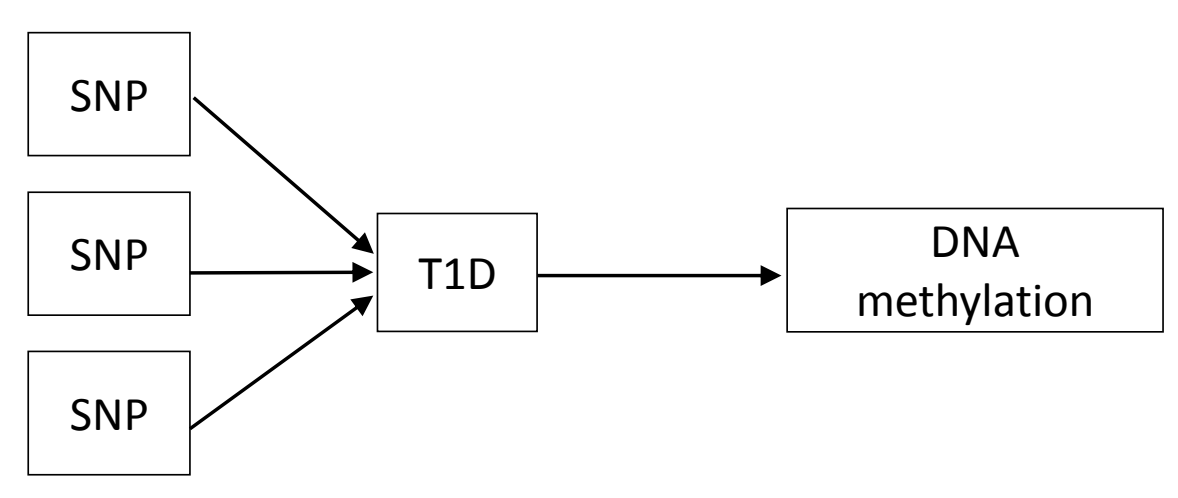

d

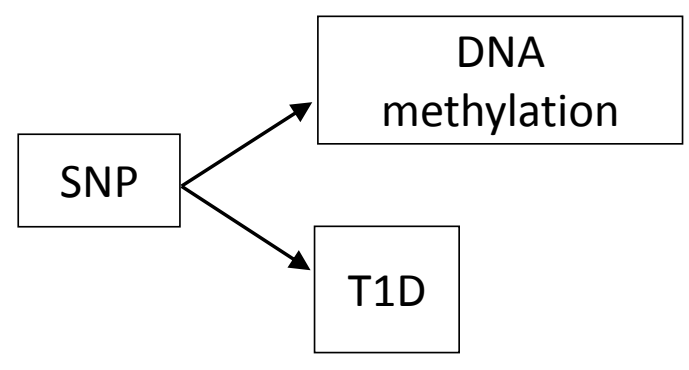

Figure 1: Four possible scenarios that could explain the associations between SNP, DNA methylation and T1D.

a, DNA methylation mediates the genetic risk of T1D; b, SNPs first increases T1D liability, which in turn changes DNA methylation levels; c, a SNP that regulates DNA methylation could simply be in LD with another causal variant that influences T1D; d, a SNP is associated with DNA methylation and T1D via independent biological pathways (horizontal pleiotropy). 


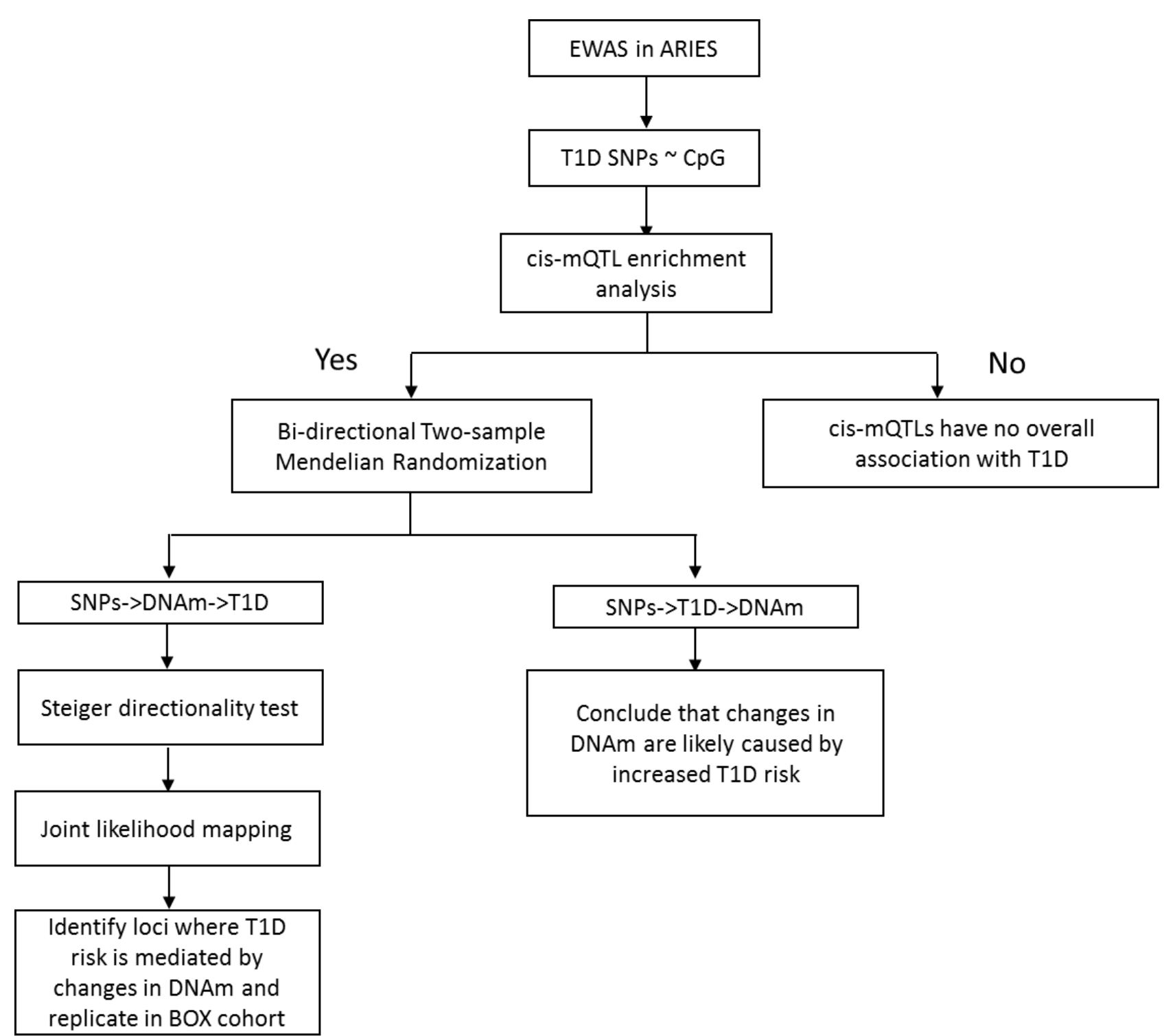

Figure 2: Flow chart summarising the overall analysis procedure in this study.

EWAS: epigenome wide association analysis; DNAm: DNA methylation; mQTL: methylation quantitative trait loci 


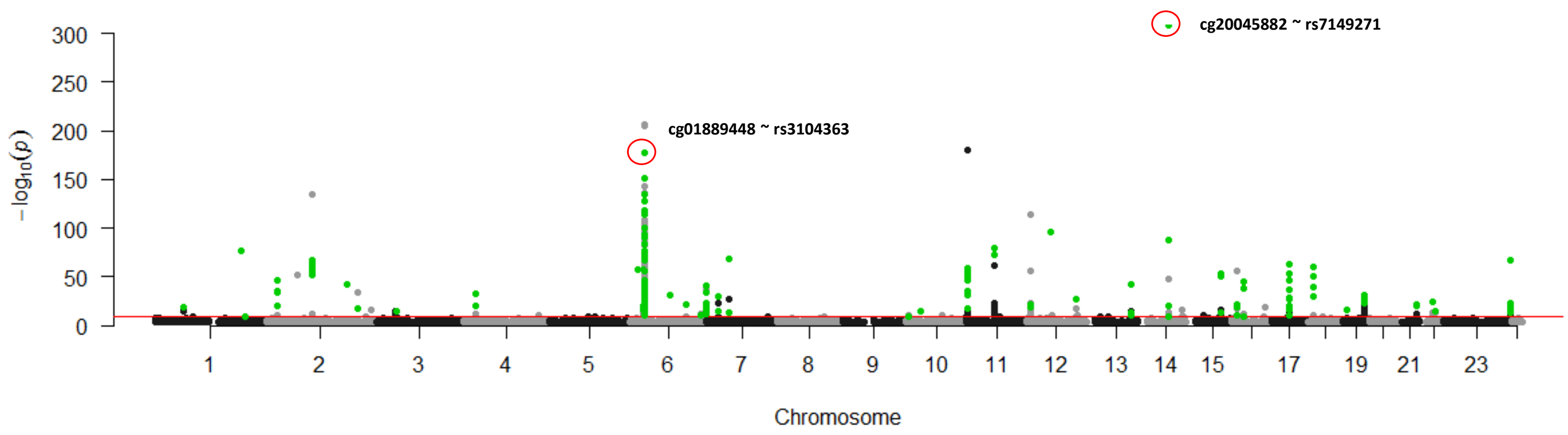

Figure 3: Genomic distribution of CpG sites that are associated with T1D GWAS variants.

Manhattan plot showing the CpG sites associated with 38 T1D GWAS variants above the Bonferroni threshold 1.6e-9 (redline); green highlighted dots were those (n=166) that were consistently detected at adolescence, childhood and birth; there is a peak reflecting mQTL-CpG association at the HLA locus (chromosome 6). 
a

$$
p=0.03
$$

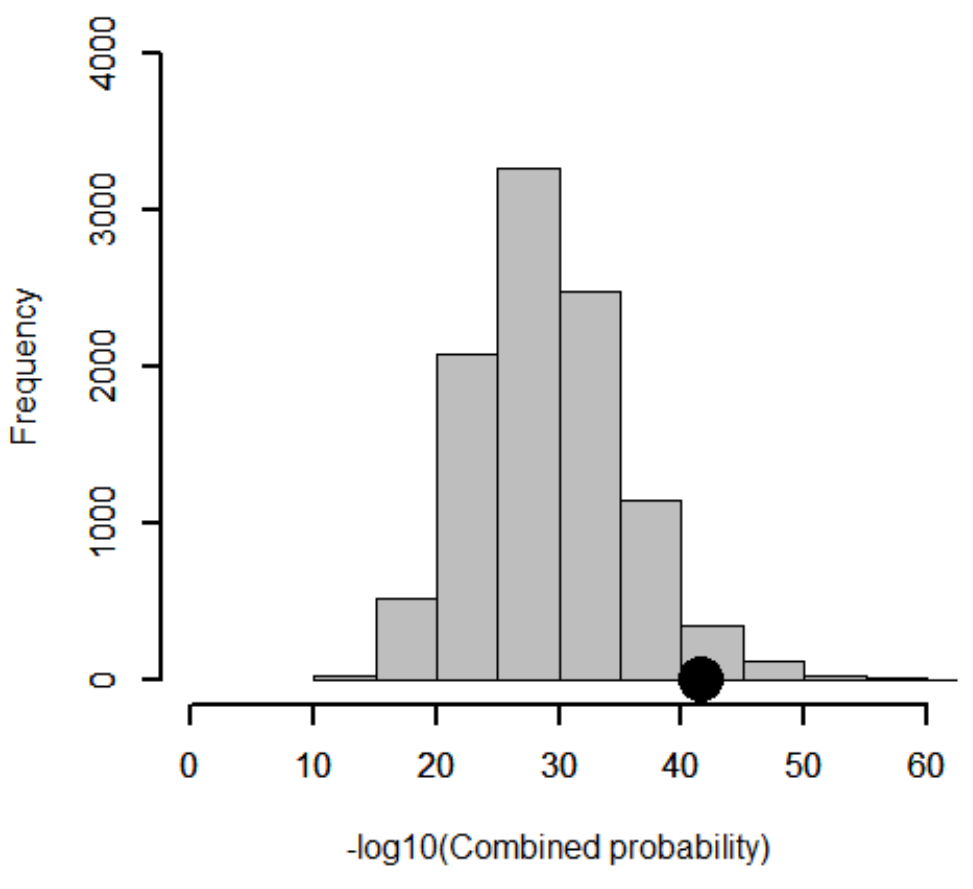

b

$$
p=0.01
$$

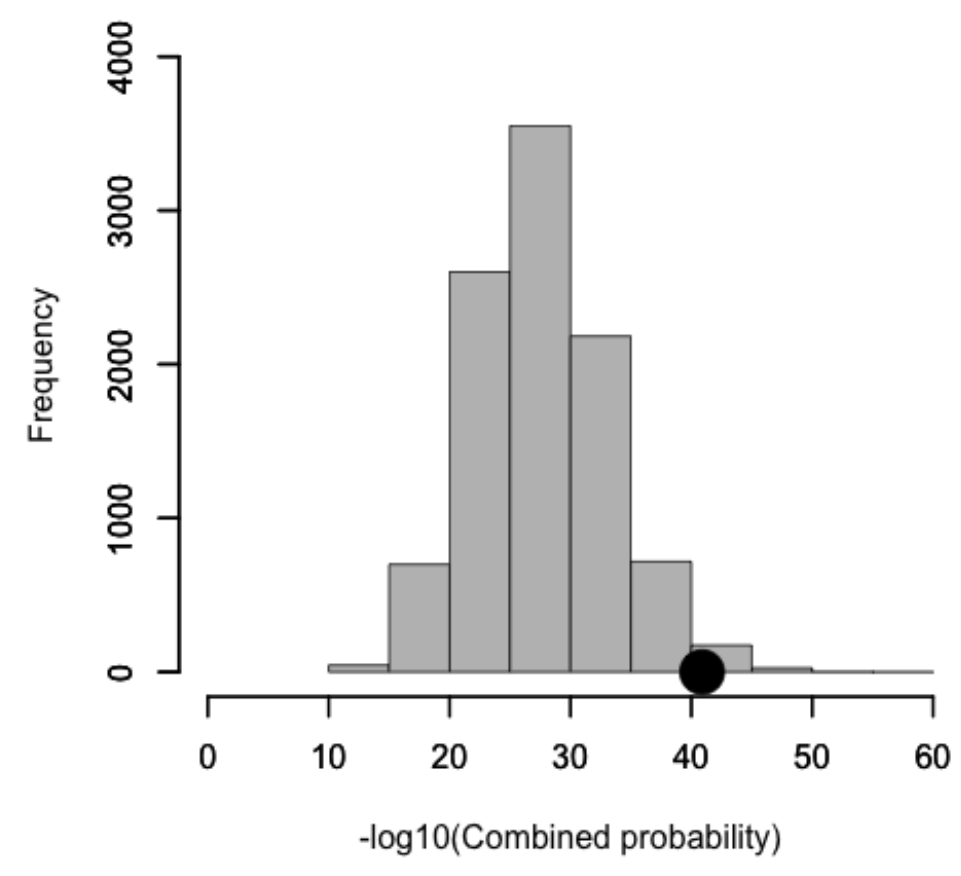

\section{Figure 4: cis-mQTLs are enriched in SNPs with low GWAS $p$-values associated with T1D.}

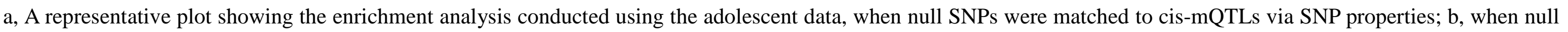
SNPs were matched to cis-mQTLs via genomic annotations. T1D GWAS $p$-values were extracted from meta-analysis Data 1. 

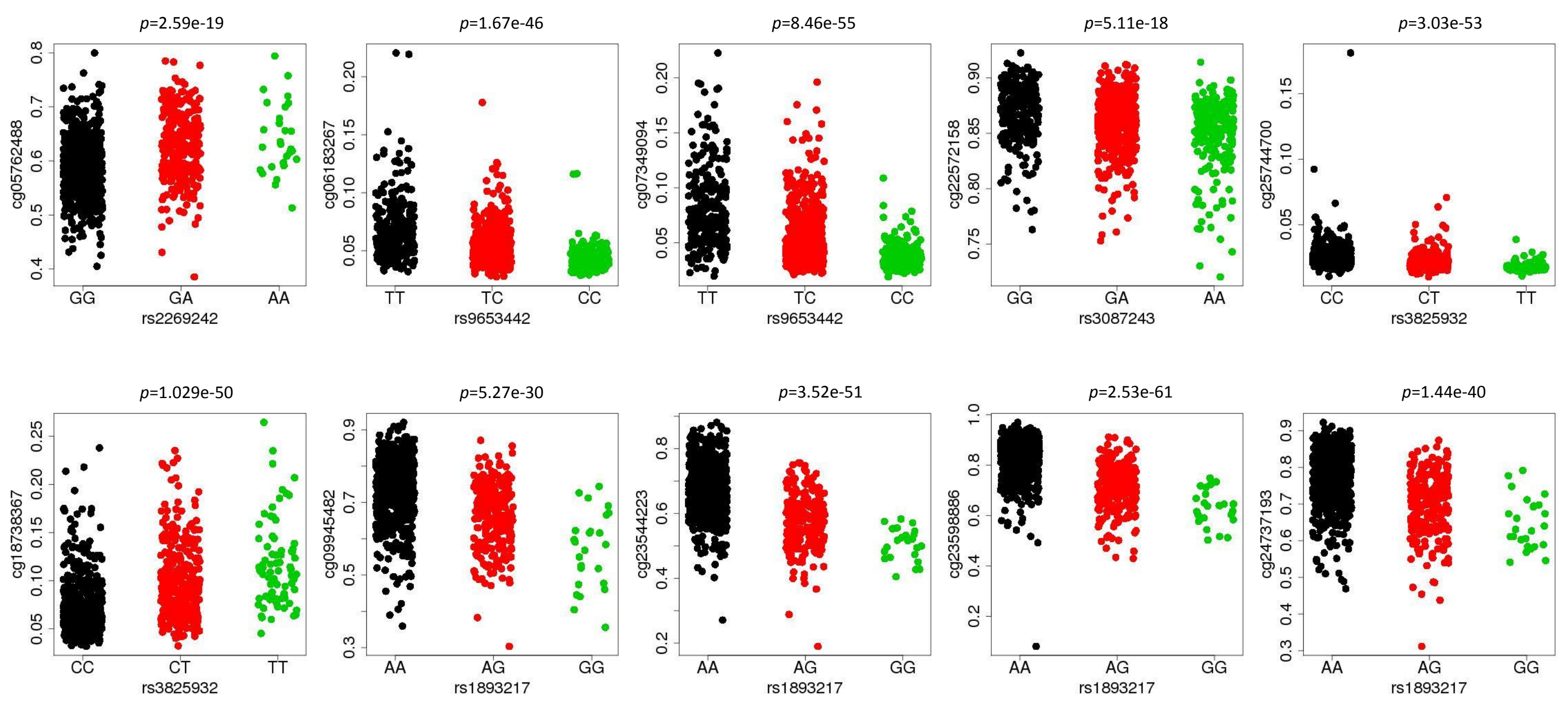

Figure 5: DNA methylation levels of CpG sites and their associations with T1D GWAS variants that survived JLIM analyses, obtained from the ARIES adolescent participants.

$\mathrm{Y}$ - axis represents beta values for each CpG site. The inner most genotype in $\mathrm{X}$ - axis is comprised of two other alleles, the outer most genotype is comprised of two effect alleles. 

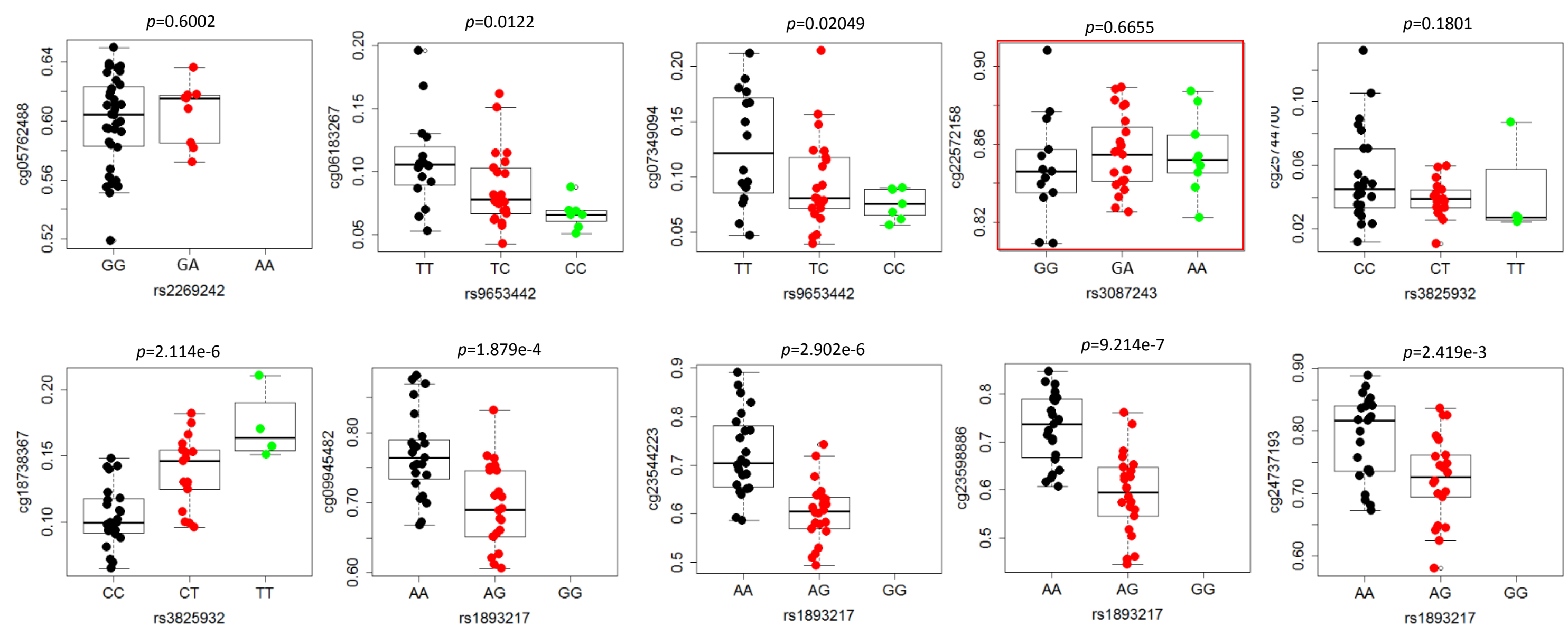

Figure 6: Replication of SNP and DNA methylation associations in the Bart's oxford T1D cohort.

45 individuals were analysed in the BOX cohort. Nine out of ten SNP-CpG pairs showed similar associations compared to the ARIES participants. The SNP - CpG pair that did not replicate the ARIES result was highlighted in red. 